\title{
Digital aroma technology for chemical sensing: temporal chemical images of complex mixtures
}

\author{
I.V.Kruglenko, B.A. Snopok*, Y.M. Shirshov, E.F. Venger \\ Institute of Semiconductor Physics, NAS Ukraine, 45 prospect Nauki, Kyiv, 03028, Ukraine \\ * B.A.S.: Email: snopok@isp.kiev.ua; Telephone: +(380) (44) 26556 26; Phone (Voice\&Fax): +380 (44) 2651827.
}

\begin{abstract}
The reliability of Electronic nose applications is mainly based on the sensitivity, repeatability and discernment properties of the sensors composing the array. Due to nature of the chosen sensitive layers, the sensors are particularly suitable to classify environments in which compounds with various chemical functionality play a key role. This article is focusing on the novel strategy aimed at the improving the quality and quantity of chemical information from the sensing instruments based on incorporation kinetic aspects of the array response for perfect formation and improved discrimination of chemical images of the probes. We describe a procedure for reducing both time response and number of elements of gas sensor arrays based on principal component analysis. Thus, digital aroma technology may be used not only as background for formation of chemical images using intelligent sensor massifs but also as an effective experimental tool for characterization and selection coatings with desired chemical functionality by using time-resolved pattern recognition.
\end{abstract}

Keywords: chemical images, electronic nose, sensors, calyxarene, response time, principal component analysis, quartz crystal microbalance, perfumes, sensor array.

Paper received 15.11.00; revised manuscript received 30.11.00; accepted for publication 12.12.00.

\section{Introduction}

In many cases an analysis of quantitative and qualitative composition of multicomponent chemical media (MCM) using the classic methods of analytical chemistry runs into difficulties. They stem from the presence in a sample of many fractions of unknown nature [1]. The traditional approach to the express chemical analysis of MCM presumes application of specific sensor sets. Each set is optimized for detection of strictly defined gas mixtures. Realization of this approach, however, is efficient only if the absolute values of concentrations are to be found from measurements. In this case it is practically impossible to obtain highly specific sensor coating that are sensitive to the specific molecules only [2]. Principal reason for this lies in presence of similar functional groups with like spatial structures and close physico-chemical properties within the same chemical class [3]. So wide application of the «a gas-a sensor» approach seems to be unlikely, taking into account that at the moment the total number of known chemical compounds is several millions.
Formally speaking, any MCM could be treated using not a sum of its components but some model with a set of parameters that is characteristic of each MCM - the socalled chemical image (CIM) [4]. Realization of such an approach involves use of a limited number $m$ of sensors with a wide dynamic range $B$ for the output signal changes. Assuming that the sensitive layers of the above devices have different chemical functionalities, one obtains that the number of possible combinations is proportional to $\left(B / B_{0}\right)^{m}$ where $B_{0}$ is the reading accuracy. To illustrate, for $m=8, B=100$ and $B_{0}=1$ the number of possible combinations exceeds the number of known chemical compounds by several orders of magnitude. Obviously correctness of the above estimation is determined, first of all, by functionality variations for the elements from sensor set, or, to put it differently, by a degree of correspondence between the variability of MCM interaction with a sensor element array and that of the MCM composition [5].

Here we present an analysis of the ways to form CIM for MCM using sensor arrays. Our analysis takes into account the chemical functionality features for the sen-

\footnotetext{
* To whom correspondence should be addressed.
} 


\section{I.V. Kruglenko et al.: Digital aroma technology for chemical sensing: temporal ...}

sor array, as well as types of the sensor response parameterization and measuring technique that are aimed at increase of the identification degree for composite chemical mixtures.

\section{Chemical functionality of multisensor systems}

\section{Direct and indirect methods for CIM formation}

A specific CIM kind is determined by the type of physicochemical characteristics of a sensitive element. Their changes that result from interaction with MCM serve for CIM formation. Among the direct methods for CIM formation chromatography and mass spectrometry [6] are usually mentioned. They detect presence of specific molecules or chemical groups in the sample studied. Those approaches that use indirect change in the output signal of a physical transducer stemming from interaction between a sensitive layer at the transducer surface and gas molecules are assigned to the indirect methods of CIM formation for MCM [7]. Realization of direct methods requires considerable expenses, as well as some specific conditions (such as vacuum and high temperatures) [8]. Contrary to this, indirect methods that are based on physical transducers of various types make it possible to develop simple, compact, completely automated devices [9].

A wide variety of physical transducers exist that are used to form multisensor arrays [10]. The following three approaches hold predominant position among them. First of all, these are the metal-oxide semiconductor (MOS) sensor systems [11]. Their operation is based on oxidation/reduction of the gas mixture components at elevated $\left(250-450{ }^{\circ} \mathrm{C}\right)$ temperatures. At present the multisensor system on MOS arrays are most extensively employed [9]. However, such factors as slight distinctions in specificity, the response function nonlinearity and high dependence on presence of water in a sample, as well as sample composition changing during the measurement, impose limitations on possibilities of their wide use.

The conducting organic polymers (COP) represent another main class of materials used for development of sensitive arrays [12]. The principal advantages of this class of sensitive elements are a possibility to modify polymer backbone using some specific peripheral groups of set chemical functionality, as well as the ease of fabrication and measurement [13]. In this case an informative signal is a change in the system conductivity. So the required sensitivity can be obtained for a limited number of molecules only. They may be donor of acceptor ones, depending on the type of the polymer backbone and peripheral substituents that increase or decrease the charge carrier concentration [14]. The composite sensitive elements of COP sensors are polymers containing intercalated conducting particles [15]. They use the effects of coating structure modification resulting from interaction with a gas environment. Their selectivity that is due to gas molecules «solubility» in the film bulk also is limited
[14]. The COP sensors demonstrate a nonlinear response and dependence on moisture presence in a sample (just as the MOS ones).

Both the above types of sensors (MOS and COP) are characterized by combination of a sensitive layer and physical transducer proper. As a result, the measurement process affects the reaction ability/specificity of the receptor centers interaction with molecules detected. A radically different approach can be realized using the mass sensitive transducers [16]. It provides separation of chemical interaction processes from their transformation into a corresponding sensor response.

There exist a wide range of the nonselective acoustic gravitometry transducers. These are quartz crystal microbalance (QCM) [17], surface acoustic wave oscillators (SAW) [18], systems based on the Love-wave [19], etc. They enable one to develop sensitive arrays for different purposes. Each such array has its own merits and demerits [20]. Among the features of piezoelectric crystal transducers using bulk acoustic waves are high sensitivity (several $\mathrm{ng} / \mathrm{cm}^{2}$ at a frequency of about $10 \mathrm{MHz}$ ) [21], possibility to form on them sensitive layers with bulk (as well as surface) receptor centers [22] and realize various measurement modes (frequency drift, Q-factor variation) $[23,24]$. That is why such transducers may be considered to be among the most promising for development of smart sensor system of a new generation, such as those of the «electronic nose»-type (EN) [25].

\section{Chemical functionality of a multisensor system}

In the mass sensitive devices (based on QCM, SAW, etc.) processes of chemical interaction and sensor response formation are separated. Therefore functionality of a multisensor system is determined by the following three factors:

- Chemical functionality of a sensor array: the identification degree for different MCM is determined by distinctions in chemical functionality [26, 27] and number of elements $[28,29]$ in the sensor array. A system of «primary odors» may be used to form an optimal sensor array, just as the system of primary colors is used to form the color scale. At present there exist several odor systems: Aristotle's system, Henicks' prism, Amoore's stereochemical theory [30], etc. They are based on an analysis of human and animal olfactory systems [31]. Thus, according to the above approaches, the sensor array optimization will form an effective sensitivity similar to the human one.

- Parameterization type for the sensor array response during CIM formation. Choice of optimal parameters that largely take into account the features of interaction between a sensitive array and MCM will determine, to a great extent, the identification degree for different MCM [32].

- A character of sample preparation and measuring technique. The latter should provide obtaining of maximal information on the interaction process [33], while the sample preparation has to provide gas content stabil- 


\section{I.V. Kruglenko et al.: Digital aroma technology for chemical sensing: temporal ...}

ity during measurements, absence of concentration constraints (especially when only the liquid sample can be open to inspection).

\section{Chemical functionalities of an individual receptor center and sensing coating as a whole}

At present there are a rather big number of methods to form thin-film coatings over a wide thickness range, from several $\mathrm{nm}$ up to hundreds of $\mathrm{mm}$ [34]. However, the new promising approaches based on the self-assembling [35] and controlled layer-by-layer deposition [36] techniques cannot provide the required range of receptor centers of preset chemical functionality for layer formation on various substrates. The reason lies in the lack of appropriate chemical technologies. At the same time some classic microelectronic technology techniques (such as thermal evaporation in vacuum followed by condensation on the corresponding substrates [37]) have been well developed for most of low-molecular organic materials and types of substrates. These techniques make it possible to get stable coatings with required physico-chemical properties.

An essential aspect of the sensitive layer formation is correspondence between chemical functionalities of an individual receptor center and a film as a whole. The OMC structural organization is complicated [38]. In fact, OMC demonstrate several levels of structural organization, namely, $(i)$ intermolecular structure that determines chemical functionality of a compound, (ii) lattice structure that determines character of packing in a solid, and (iii) macroscopic structure that affect the mechanisms for energy, charge and matter transport. The chemical functionality of sensitive layers is determined by the OMC electronic structure; so it also depends on the above features of structural organization. However, due to a rather weak intermolecular interaction in $\mathrm{OMC}$, its molecules retain their individualities to a great extent. Therefore the electronic structure of molecules serves as the main factor that determines both character and strength of interaction between the molecules of OMC and gas.

\section{Physical basis for intermolecular interactions}

Within the local molecular approach the strength and specificity of intermolecular interaction are determined by a number of factors, such as: (i) molecular size and symmetry; (ii) polarizability of the molecular skeleton; (iii) substituents type, number and polarity; (iv) degree of freedom of individual molecular components; $(v)$ hydrophilic-hydrophobic properties of interacting components, etc. Depending on the features of electronic structure of molecules, the energy of intermolecular interaction with gas molecules may vary by several orders of magnitude - from several thousandth $\mathrm{eV}$ to several $\mathrm{eV}$ [39]. At the same time a typical value of a covalent binding energy lies within a rather narrow (from 2 to $4 \mathrm{eV}$ ) energy range. This results from the fact that an act of intermolecular interaction is due to a combined effect of processes of different physical nature. The higher the interaction specificity, the stronger is the interaction. In the limiting case of chemical adsorption a strong chemical bonding of the exchange type is formed. We are interested, first of all, in those processes that are reversible at normal conditions (physical adsorption). In this case an adsorbed molecule is held on the solid surface by the forces of predominantly electrostatic nature. These are van der Waals, hydrogen and donor-acceptor interactions.

The van der Waals (vdW) forces are a special kind of weak nonvalent interaction. The most general type of these forces is dispersion force. The dispersion forces are responsible for attraction between nonpolar electrically neutral molecules [40]. Due to a coherent interaction between the nonstationary fields of quantum fluctuations of the electron density distribution for outer (i.e., most weakly bound) $\sigma$ - and $\pi$-electrons in molecules, the average interaction potential value is nonzero. This results in appearance of attraction forces. Thus the dispersion intermolecular interaction may be treated as a version of electronic polarization [41].

One of the main features of the dispersion interactions is that they are not oriented somewhere. This is due to the fact that optical polarizabilities far from resonance fall within the integral molecular properties. This means that they do not depend on the energy spectrum features but are determined by the chemical bonds in total. In this case one may relate a vdW surface to a molecule, and this surface will determine the intermolecular interactions features. The minimal total potential energy of interaction is determined by the complementarity condition for the molecular vdW surface at maximal number of the «atom-atom» contacts [42]. From the phenomenological viewpoint one may describe the total potential energy of the receptor/analyte interaction with a set of model force fields that involve the energies of bond stretching, torsion distortions, electrostatic interaction, etc. [43]. Such an approach that is based on the methods of molecular mechanics serves as a foundation for theoretical approaches to determination of the driving forces for the substrate-receptor interaction. The most prominent examples of such receptor centers are concave or cavity host molecules, such as calyxarenes [44], resorcinarenes [45], cyclodextrins [46], etc. It should be noted that according to the Amoore's stereochemical theory [30], five of the seven primary odors have receptor centers of just the affine type.

Polar molecules (those having fixed dipole moments) demonstrate interaction not only with the dispersion forces but also with dipole and induction forces of electrostatic origin [47]. In this case, if a molecule has a fixed dipole or quadrupole moment, then the interaction strength depends also on momentum orientation. Therefore the electrostatic attraction forces tend to orient molecules. So this type of the vdW forces is known also as the orientation interaction. The vast majority of odoriferous substances have non-zero dipole moments [48]. So the interaction of just this type may determine in what way the ligand-receptor interaction processes proceed. 


\section{I.V. Kruglenko et al.: Digital aroma technology for chemical sensing: temporal ...}

Another type of intermolecular interaction that is characteristic of the reversible adsorption processes is hydrogen bonding (HB) [49]. The HB energy is much over the $\mathrm{vdW}$ one. Therefore it determines a stronger interaction of higher specificity. HB may be formed by various interactions that are of classic (Coulomb), as well as quantum-mechanical, nature. So HB energy and other properties may vary over wide ranges, depending on a specific $\mathrm{HB}$ type. $\mathrm{HB}$ formation may serve an example of a nucleophilic interaction when a ligand-receptor complex is formed to which one of the receptor types corresponds, according to Amoore [30].

A weak donor-acceptor bonding (DAB) also can be a reason for physical adsorption (weak charge-transfer complexes or contact complexes) [50]. A specific feature of $\mathrm{DAB}$ is partial electron transfer from a donor to acceptor even in the ground state. A critical factor in this case is that interactions of different nature (Coulomb, polarization and resonance) take part in DAB formation. Generally the DAB energy is the biggest of the above ones. It determines those features of electrophilic interaction that correspond to the seventh principal type of receptor centers for molecules with high electron affinity, according to the Amoore's stereochemical model [30].

In conclusion it should be noted that the character of forces responsible for the receptor/analyte complex formation determines not only the interaction process specificity and reversibility (they are characterized by a number of molecules held by the sensitive layer). It also determines the kinetic features of the reaction of bound complex formation. Therefore the kinetic characteristics of the physical transducer response may contain important information that can serve to a more complete formation of CIM for the mixtures analyzed.

\section{Perfume formulation}

The perfumes (i.e. éau-de-Cológne) involve: (i) an alcohol-water base; (ii) a stabilizer (such as diethyl phthalate); (iii) a perfume composition that may contain extracts of natural fragrant substances, as well as synthetic products. The so-called essential oils [51, 52] are most often used as fragrant substances. From the chemical standpoint they are hydrocarbons or their ordinary derivatives. Generally, for a substance to be perceived by the human olfactory system as fragrant, it has to [53]: $(i)$ have molecular mass over 10-20 but below $300 \mathrm{amu}$; (ii) be of a characteristic size that is not over several nm; (iii) involve unsaturated bonds and (iv) specific chemical groups; (v) have a moderate diffusion rate and high volatility in normal conditions.

It was noted earlier that chemical functionality of a sensor array as a whole is the governing factor in successful identification of composite mixtures. Both affine-type and nucleo/electrophilic receptor centers are required to perform a successful analysis. According to the above, we chose as sensitive elements those organic compounds that represent predominantly affine receptors (calyxarenes $C[4] A, C[6] A, C[8] A)$ and «redox»-centers (Pent, TCT,
TTT). The internal (external) cavity size for calyxarene molecules of affine centers were from $0.63(1.36) \mathrm{nm}$ for $C[4] A$ to 0.79 (1.8) nm for $C[8] A$ [54]. These values span the ligand size that are characteristic of the perfume molecular elements. In our studies we used molecules Pent and TTT as electrophilic centers. These molecules have low-energy molecular orbitals and high polarizability $[55$, 56]. To make allowance for possible nucleophilic interactions of the donor-acceptor type, TCT was included to the sensor set [57].

\section{Chemosensory system based on microbalance transducers}

\section{Physical principle of measurement}

In the sensors based on the piezoelectric transducers a dependence of the resonance frequency $f$ of acoustical resonator on the mass $\Delta m$ of a substance at the sensor surface is used to detect intermolecular interactions. Very often dependence between the resonance frequency variation $\Delta f$ and $\Delta m$ is assumed to be linear [58]. In fact this assumption is valid only for a rather ideal case when both density and viscosity of the sensitive layer are the same as the corresponding parameters of the transducer and do not change during its interaction with an analyte [59]. The second of these assumptions is not true in many cases and may distort the actual proceeding of the process [60]. If, however, one can neglect the hydrodynamic interaction (elastic-viscous relaxation) between the physical transducer and environment (this is true mostly for measurements in a gas phase) and consider the sensitive layer as rigid, then the variation $\Delta f$ of the piezoelectric transducer frequency due to the mass change $\Delta m$ on its surface is of the following form [61]:

$\Delta m=-S \cdot \frac{\Delta f}{2 \cdot f_{0}^{2}} \cdot Z_{c q}$

Here $S$ is the electrode area; $Z_{c q}$ is the specific acoustic impedance of the quartz crystal; $f_{0}$ is the unperturbed fundamental resonance frequency of the piezoelectric element. In the case of a thin rigid mass layer without liquid contact, the equation (1) equivalalent to the wellknown Sauerbrey's equation [62]:

$\Delta f=-C_{Q} \cdot \Delta m / S$.

Here $C_{Q}$ is the QCM integral sensitivity to additional mass on the surface.

\section{Static and dynamic methods for formation of MCM chemical images}

In the case of piezoelectric transducers the adsorption process can be directly characterized with the resonance frequency change values (at different moments) and their 


\section{I.V. Kruglenko et al.: Digital aroma technology for chemical sensing: temporal ...}

time derivatives, as well as various combinations of the above quantities. One should specially note that use of approaches based on experimental curves fitting with analytical functions to obtain the response parameters seems to be inappropriate in this case. The reason is that very often the simple kinetic models for adsorption cannot adequately describe the curves for the sensor array response, because of MCM components diffusion into the sensitive coating bulk [64], change of its structure [65], uncontrolled fluctuations of temperature and pressure. Indeed, if one uses for MCM CIM formation only stationary quantities (i.e., those that characterize a system after an equilibrium has established), then one may omit important information concerning character of interaction between highly volatile components with different mobilities/activities and the sensitive layer. And the sensitive layer prehistory strongly affects the features of the receptor/analyte interaction in the initial portion of the kinetic curve. Thus the problem how to choose an optimal parameter that could take into account the kinetic and stationary features of the sensor array response, as well as some leveling effects of the pre-starting procedure, is of importance for both fundamental science and practical applications.

The normalized area under the kinetic curve may serve as a parameter accounting for both kinetic and equilibrium features of adsorption process. To this end one can also use the so-called $i V$-parameter that depends on the interval of integration $t^{\text {th }}$ :

$i V\left(t^{t h}\right)=\frac{1}{t^{t h}} \int_{t=0}^{t=t^{t h}} n(t) d t$

Let us consider the case of monomolecular Langmuir adsorption [66]. Then

$n(t)=N \cdot[1-\exp (-a t)]$

where $a=\alpha P+\beta ; b=\beta / a ; N=\frac{N^{*}}{1+b / P} ; \alpha=\frac{\chi s}{\sqrt{2 \pi M k T}}$

$\beta=v \exp (-q / k T)$. Here $n$ is the number of gas molecules held at the unit surface area at the moment $t ; N^{*}$ is the number of adsorption centers; $M$ and $s$ are the adsorbed molecule mass and area, respectively; $\chi$ is the sticking coefficient; $v$ is the desorption probability; $q$ is the binding energy of a particle on the adsorption center. If the initial condition is $n=0$ at $t=0$, then:

$$
i V\left(t^{t h}\right)=\frac{1}{t^{t h}}\left\{N \cdot t^{t h} \cdot\left(1+\frac{1}{a \cdot t^{t h}} \cdot \exp \left(-a \cdot t^{t h}\right)-\frac{1}{a \cdot t^{t h}}\right)\right\} .
$$

It is notable that at small $t^{\text {th }}\left(t^{\text {th }}<<1 / a\right)$ one obtains

$i V\left(t^{t h}\right) \cong \frac{1}{2} \cdot \frac{N^{*}}{1+b / P} \cdot a t^{t h}$
This expression contains information on both the process kinetics (parameters $a$ and $b$ ) and adsorbability of the surface $\left(N^{*}\right)$. When $t^{\text {th }} \rightarrow \infty$, then the $i V$-parameter (as well as $n$ ) goes asymptotically to $N$. One can easily calculate the $i V$-parameter from experimental data. Thus the use of this parameter allows one to take into account the characteristic features of interaction process. At the same time separation of the most specific features is determined by the choice of the interval of integration $t^{\text {th }}$. An additional argument for the use of $i V$-parameter for sensor response analysis is the fact of the signal-to-noise ratio increase when calculating this parameter. This is due to leveling of the stochastic noise (whose time average value is zero).

\section{Methods and materials}

\section{QCM devices and electronics}

A multisensor QCM-based analyzer involves the following units: (i) a flow-type chamber with sensor array; (ii) a quartz oscillator unit; (iii) a unit for frequency measurement with RS232 interface based on a dedicated microprocessor (AT89C2051); (iv) a gas mixtures generator; (v) a computerized system to collect and process information. To provide vapor-gas mixtures stability (if the sample could be supplied in liquid state only), a mechanical agitator maintained a constant speed of the gas carrier (air) that was passed through the sample volume. The test oscillators were connected to the frequency meter in series; time for measurement was $1 \mathrm{~s}$ and the frequency reading accuracy being $1 \mathrm{~Hz}$. We did not use an additional thermal stabilization of the system, as well as control over gas carrier humidity and common pressure. The

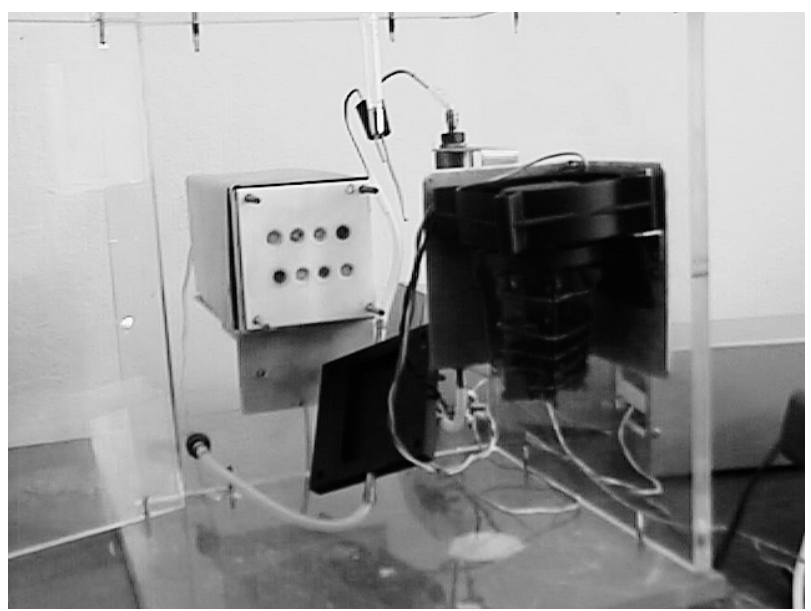

Fig. 1. General view of the components in the quartz crystal microbalance instrument. The central element is the sensor array mounted on the main frame inside the thermostatically controlled chamber. 


\section{I.V. Kruglenko et al.: Digital aroma technology for chemical sensing: temporal ...}

general view of the experimental plant is shown in Fig. 1.

The AT-cut (RK169) crystals [63] were used as physical transducers. They operated in the shear vibration mode; the fundamental frequency was $10 \mathrm{MHz}$. The quartz plates were fixed with silicone sealant (along the perimeter) in Teflon cases. They provided access of mixture studied to only one of the quartz transducer faces. The sensors made in such a way did not demonstrate changes in the basic resonance frequency (as compared to the standard wire fixing). The advantages of the above fixing reveal themselves, however, at abrupt changes of the gas-carrier flow, since the design used provides stable oscillation by QCM even in such conditions

The measuring procedure involved the following stages: air circulation until the transducer frequency is stabilized $( \pm 5 \mathrm{~Hz})$; vapor-gas mixture circulation; blowing down with pure air until the QCM frequency returns to its initial value. To provide the same conditions and exclude the effect of previous experiments, we blew hot (temperature of about $60^{\circ} \mathrm{C}$ ) air through the measuring chamber for $15 \mathrm{~min}$ after every measurement. This procedure has always retained the sensor operational characteristics. The number of measurements was not below five. We did not, however, average the results obtained, since such a procedure runs counter to that of the multisensor system application. The response distinctions for different sensors did not exceed $10 \%$. They were due to fluctuations in the gas-carrier (air in the working area) composition and flow rate.

\section{Sensitive layers and the samples used}

Calyxarenes, tret-butylcalyx[4]arene $(C[4] A)$, tretbutylcalyx[6]arene $(C[6] A)$ and tret-butylcalyx[8]arene $(C[8] A)$ were kindly given to us by Prof. V.I. Kal'chenko (Institute of Organic Chemistry of NASU, Kyiv). Linear polyacenes, namely, pentacene $\left(\right.$ Pent, $\left.\mathrm{C}_{22} \mathrm{H}_{14}\right)$ and substituted tetracenes (tetrathiotetracene (TTT, $\mathrm{C}_{18} \mathrm{~S}_{4} \mathrm{H}_{8}$ [56]) and tetrachlorotetracene $\left(T C T, \mathrm{C}_{18} \mathrm{Cl}_{4} \mathrm{H}_{8}\right.$ [57])) were kindly given to us by Prof. Ya.I. Vertsimakha (Institute of Physics of NASU, Kyiv). All the above substances were used without an extra purification.

Both calyxarene and linear polyacene films were prepared by thermal sputtering onto the metal electrodes of QCM (on one side). The above procedure was made in vacuum (VUP-5M, residual vapor pressure of about $\left.5 \cdot 10^{-4} \mathrm{~Pa}\right)$; temperature $(297 \pm 2 \mathrm{~K})$. The mean condensation rate was $0.1 \mathrm{~nm} / \mathrm{min}$. During film sputtering their thickness was monitored with a quartz thickness gage and was about $100 \mathrm{~nm}$. The transducers with deposited sensitive layers were placed in the measuring plant and exposed to ethanol vapor (in standard conditions) until a reproducible response of the whole sensor array was achieved. This required from 10 to 15 cycles. Such a prestarting procedure enabled to stabilize parameters of thin films [67]. After this the sensitive element array was used to perform the planned measurements.

The following three types of perfumes (GOST 1723671) served as test samples: éau-de-Cológne «Slavutich» (group «Extra», produced by «Alye Parusa», Nikolaev); éau-de-Cológne «Consul» (group «Extra», produced by «Soyuzparfumerprom and Marbel», Moscow-Paris); éaude-Cológne for children «Antoshka» (group B, produced by KhPKF, Kharkov). The sample volume was $12 \mathrm{ml}$ in every case.

\section{Mathematical processing of sensor response.}

According to the above approach, we used the following three parameters to form CIM: the sensor frequency change rate $\Lambda$, saturation level $\Delta f_{\max }$ and $i V$-parameter. The parameter $\Lambda$ was calculated from the initial linear portion of the kinetic curves for time values below $300 \mathrm{~s}$. The saturation level was determined by averaging the experimental values over the 4000-4800 s range. The $i V$ parameter was calculated at different time values $t^{\text {th }}$ by numerically integrating the experimental curves followed by division of the integral obtained by $t^{\text {th }}$.

For qualitative presentation of CIM of mixtures we used circle diagrams [68] whose radial axes corresponded to different sensors $(C[4] A, C[6] A, C[8] A$, Pent, TTT, $T C T)$. Point position on axes corresponded to the $\Lambda, \Delta f_{\max }$ or $i V\left(t^{t h}\right)$ values for a defined sensor.

We applied the standard method of multidimensional statistic, the principal component analysis [69-71], for comparing the response pattern. The essence of this method is a transition from the initial system of parameters (which are, as a rule, strongly correlated) to new factors that are no longer correlated. Their number is below that of the initial parameters, and their variability is the same as that of the initial parameters (or, at least, contains the maximally possible part of the latter). We performed an analysis using the statistical packet S-PLUS 2000 (MathSoft Inc.); to calculate cross correlations, we used a covariance matrix. The input matrix involved three samples («Slavutich», «Antoshka», «Consul» with one or three observations per each sample), and six $i V$-parameters (variables) that corresponded to the responses of the sensor array elements at a given cut-off time $t^{t h}$. The discrimination power for the first principal component $P C 1$ was estimated using the following contrast function:

$$
\begin{aligned}
& \Xi=\frac{\left[P C 1^{S L V} ; P C 1^{A N T}\right]-\left[P C 1^{A N T} ; P C 1^{C N S}\right]}{\left[P C 1^{S L V} ; P C 1^{C N S}\right]}= \\
& =2 \cdot \frac{\left[P C 1^{S L V} ; P C 1^{A N T}\right]}{\left[P C 1^{S L V} ; P C 1^{C N S}\right]}-1 .
\end{aligned}
$$

Here the brackets denote a length of the corresponding segment on $\mathrm{PCl}$ in absolute values.

\section{Results and discussion}

\section{General kinetic features of the sensor response}

The experimental frequency variation for the array of sensitive elements versus time curves taken for three per-

$S Q O, 3(4), 2000$ 


\section{I.V. Kruglenko et al.: Digital aroma technology for chemical sensing: temporal ...}

fumes are shown in Fig. 2. An analysis of the sensor array response made it possible to note the following principal regularities in the kinetic curves behavior: $(i)$ for all the sample types these curves are monotonic with flattening out at about $1000 \mathrm{~s}$; (ii) both absolute values of frequency variation and its growth rate for individual sensors are determined by the sample type; (iii) for sensors with sensitive coatings based on $C[6] A, C[8] A$, the signal magnitudes are maximum; contrary to this, the sensors modified with $C[4] A, T T T$ demonstrate small responses; (iv) ordering of the sensor responses in accordance with their magnitudes changes with time in different ways for different perfumes; $(v)$ transition to the state of equilibrium is achieved during different times for different perfumes; (vi) usually the kinetic curve has at least two different portions, namely, fast (until about 500 s) and slow ones; (vii) there are some peculiarities in the case of $C[6] A$, namely, the slowest growth of sensor response at adsorption, as well as a pronounced double-stage relaxation during the reverse process.

Thus a qualitative analysis of the observed responses of the multisensor system evidences that each of the perfumes studied has its own specific characteristics - signal magnitude and kinetic peculiarities.
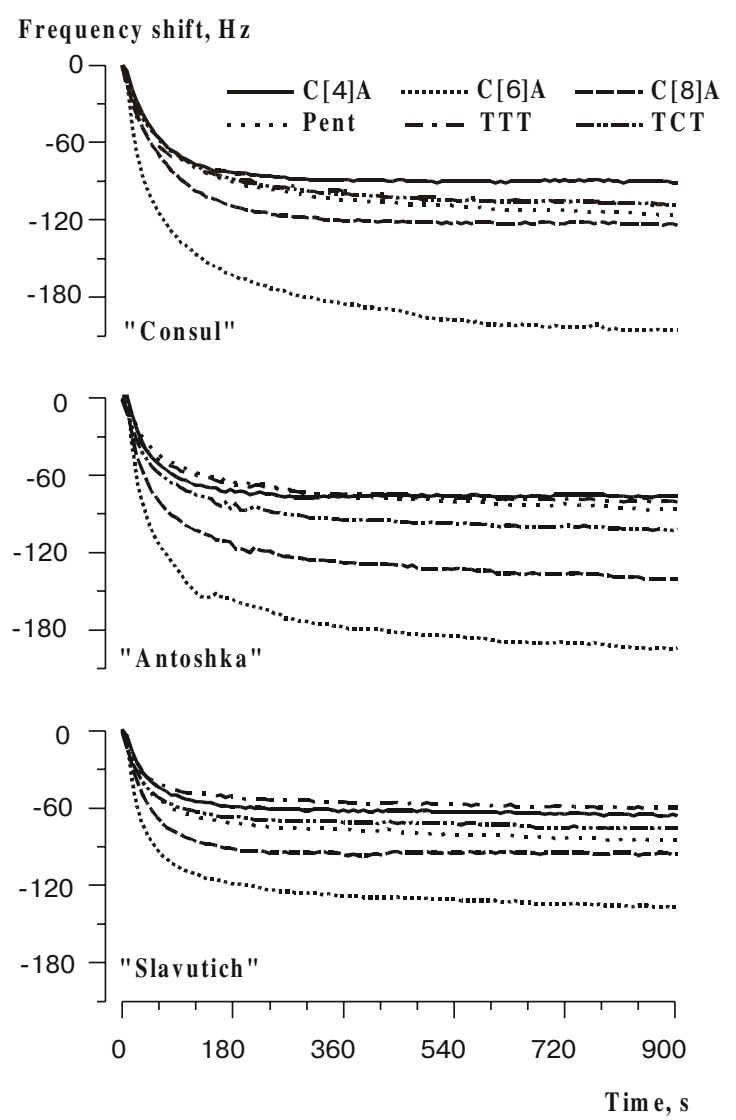

Fig. 2. Response curves for three exposures of a $C[4] A, C[6] A$, $C[8] A$, Pent, TTT and TCT - coated QCM sensors to sample outgassing for éau-de-Cológne «Slavutich», «Anthoshka» and «Consul».

\section{CIM qualitative presentation - «chemical fingerprints»}

Initially we made a qualitative comparison between the sensor array responses (in the form of circular diagrams) for different sample types using the frequency change rate $\Lambda$ and saturation value $\Delta f_{\text {max }}$ as parameters to form CIM. Thus, if CIM was formed using parameter $\Lambda$, then we could not distinguish between samples (Fig. 3a), whereas at $\Delta f_{\max }$-parameterization CIM formation require plenty of time (Fig. 3b). Since the use of $\Lambda$ or $\Delta f_{\max }$ did not enable us to correctly identify all three samples or otherwise undesirable, we calculated the $i V$-parameter at different $t^{\text {th }}$ values for sensor response parameterization and CIM formation. The results obtained are presented in Fig. $3 \mathrm{c}$ for $t^{\text {th }} \approx 330 \mathrm{~s}$. A comparison analysis enabled us to conclude that using of the $i V$-parameter has a pronounced effect on an identification degree for different sample types at small times. To illustrate, at initial (less than $50 \mathrm{~s}$ ) $t^{\text {th }}$ values the situation is just as that when $\Lambda$ was used for CIM formation. But for cut-off times mote than $50 \mathrm{~s}$ it was possible to separate all three CIM. This qualitative result supports the above supposition that concurrent allowance for both kinetic and stationary parameters at the optimal $t^{\text {th }}$ choice can substantially improve the identification degree for different sample types.

\section{Quantitative description based on principal component analysis}

To make a quantitative description of the identification procedure, we applied the method of the principal components. This method makes it possible to describe (without data structure changing) system behavior using an orthogonal coordinate system of lower dimensionality [72]. In this case the coordinate axes (principal components or eigenvectors) are a linear combination of the starting variables (criterions, here sensor responses). The coefficients, or loadings, allow for criteria relative contributions to every eigenvector. For instance, a set of data in a hyperspace projected onto the plane of the first two principal components serves as a convenient presentation of multidimensional data for a direct analysis [73]. It also provides a way to retain maximal information at a two-dimensional representation. In the methods of the principal components a coordinate system of lower dimensionality is constructed which provides the best simulation of the total set of data. So it should be stressed that it is impossible to find a unique solution (based only on an analysis of the corresponding loadings in eigenvectors) to the problem of discrimination properties of both criteria and their optimal set for solving a specific classification problem [74].

Fig. 4 shows positions of three perfumes (for one observation for each sample) at different moments in the discriminant space. Taking into account that the eigenvectors were calculated for a set of the $i V$-parameters for different $t^{t h}$ values, they change with $t^{t h}$. So relative position of perfumes that corresponds to different moments 
I.V. Kruglenko et al.: Digital aroma technology for chemical sensing: temporal ...
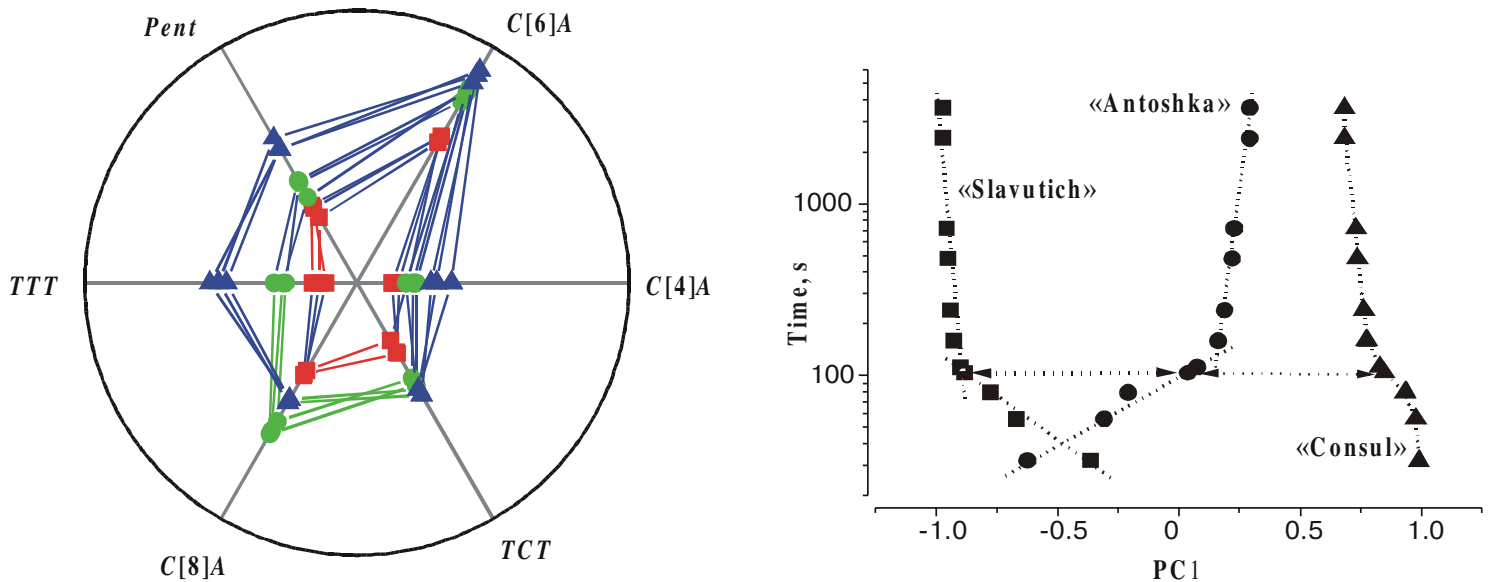

$\Delta f_{\text {max }}$ parametrization
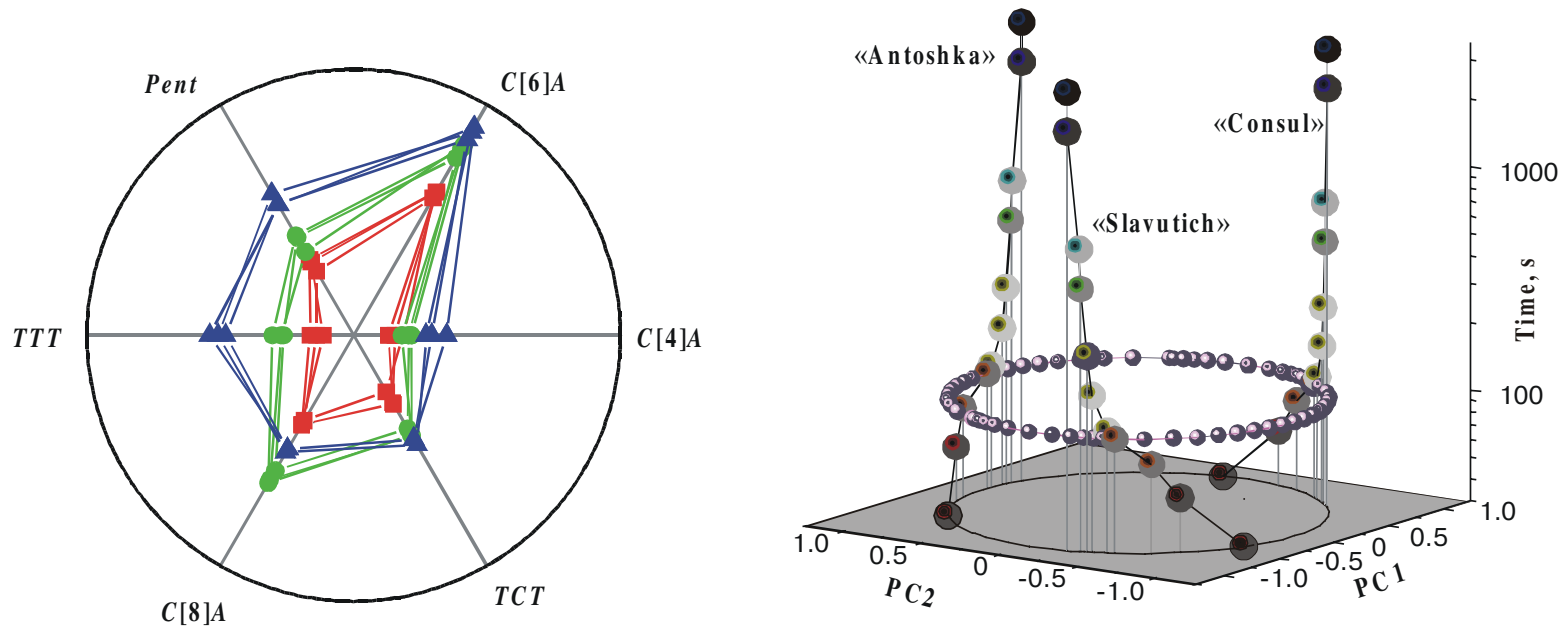

$\Delta f_{\text {max }}$ parametrization

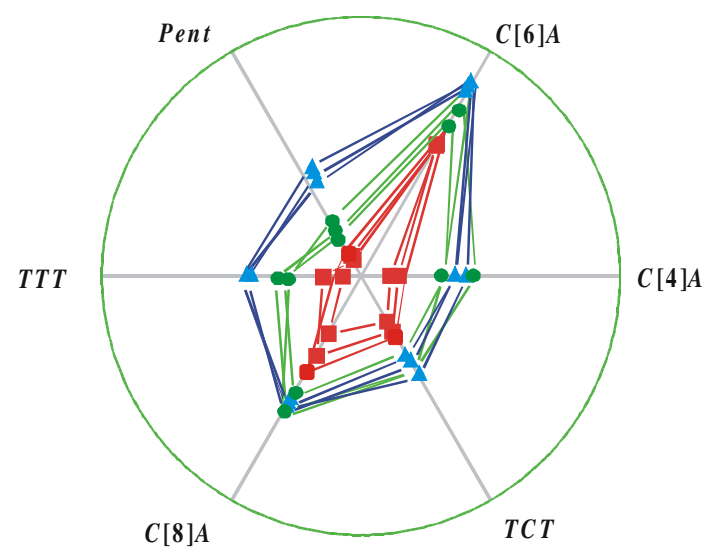

$i V$-param eter

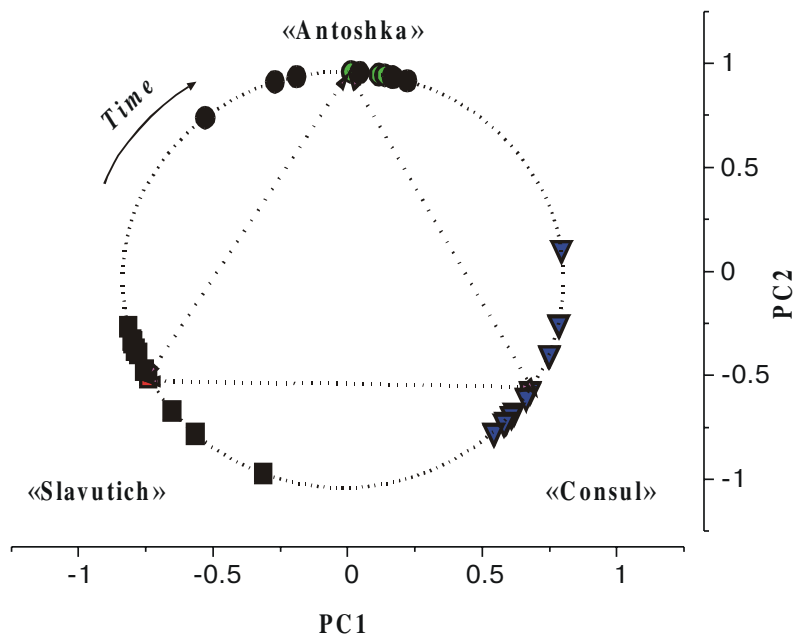

Fig. 4. Discriminant subsets (observations for three perfumes) at different moments in the principal components space. The arrows connect the points corresponding to the optimal threshold time $t^{\text {th }}$ for the «best» discrimination ability of analysis. The optimal time comply with the bumping of observations in the discriminant plane. 


\section{I.V. Kruglenko et al.: Digital aroma technology for chemical sensing: temporal ...}

should be treated as a qualitative illustration only. The dispersion (or information) that retains on the plot for eigenvector under the above transformation of multidimensional data (a two-dimensional representation of the initial six-dimensional space) at various moments is presented in Fig. 5. One can see that the two-dimensional representation gives a highly accurate (error does not exceed $1 \%$ ) description of the complete set of sensor data, that is caused by the fact that only one observation was used for each samples for the model calculations. At the same time the general peculiarities inherent real situation with noise in the data may be revealed in such analysis. For example, Fig. 5 shows that simulation abilities of the first two principal components change with time. The information presented by the first component goes down initially to about $75 \%$ at $\sim 100 \mathrm{~s}$; after this it increases up to $85 \%$ and for time values over $600 \mathrm{~s}$ remains constant. To determine the reasons for such a dependence of simulation abilities of the first principal component (that corresponds to direction of the highest data variance), one should analyze the loadings of different sensors on the eigenvector with the highest eigenvalue, depending on the measurement time.

In our calculations we used the covariance (and not correlation) scaling. Therefore it seems quite natural that the biggest contribution to the first principal component comes from $C[6] A$ that is characterized by the highest response. At the same time TTT (whose response is almost the lowest) also has high load on the first principal component. Therefore, from the standpoint of understanding the system behavior features, one should explain chemical validity of eigenvectors by analyzing their loadings. Shown in Fig.6 is the dependence of different sensors loadings on the first eigenvector. One can distinguish between the following two principal regions: a region (less than $500 \mathrm{~s}$ ) where the loadings change and that characterized by a quasistationary load value. An additional classification of sensor responses can be made from the kinetic curve form. It is as follows:

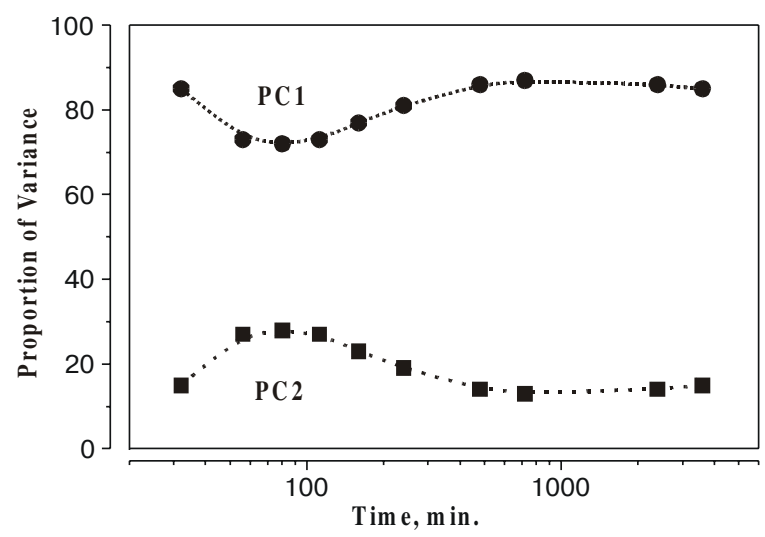

Fig. 5. The dispersion (or information) that retains on the plot for the first (PC1) and second (PC2) principal components under the transformation of the initial six-dimensional space of data observations to a two-dimensional representation at various moments during adsorption process.

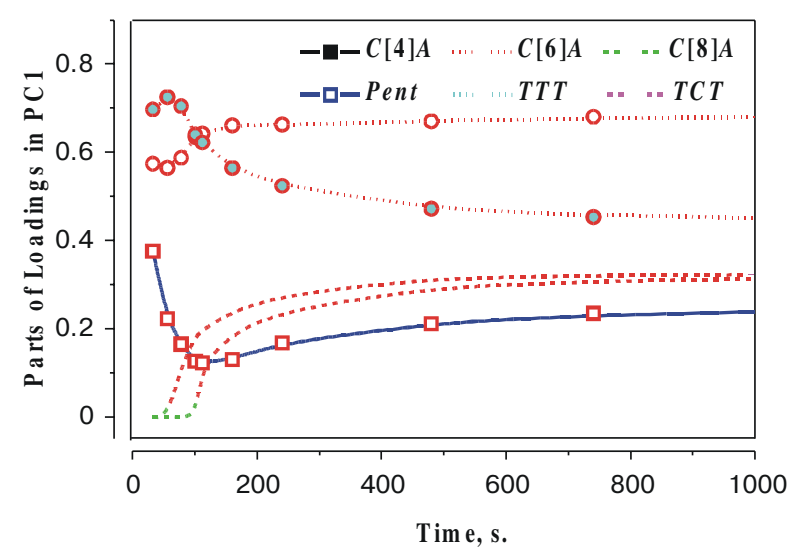

Fig. 6. The dependence of loadings (contribution from different sensors in PC1) versus adsorption time. The data matrix contains $i V$-parameters for one observation for each samples calculated for different threshold time $t^{\text {th }}$.

- sensors that practically do not contribute to the first principal component in the initial region $-C[8] A$ and $T C T$; their responses are essentially the same during the first minutes for all three samples;

- sensors whose loads on the first principal component change monotonically $-C[6] A$ (grows) and $T T T$ (drops); their responses are characterized by a rather slow and monotonous increase of the output signal from sensor element, presence of long-term response components and extreme response magnitudes - the highest for $C[6] A$ and the lowest (except the éau-de-Cológne «Consul») for TTT;

- sensors whose contributions to the first eigen-vector have extrema $-C[4] A$ (maximum) and Pent (minimum); their responses are characterized by big distinctions in the response growth rates for the samples studied.

Thus a straightforward analysis of the kinetics of loadings on the corresponding eigenvector enables one to optimize the sensor set from the standpoint of the features of its use in kinetic or stationary mode. To illustrate, the sensors with TTT (and, to a lesser degree, Pent, $C[4] A$ and $C[6] A$ ) sensitive coatings could be applied validly to analysis in the kinetic mode too, while those modified with $C[8] A$ and $T C T$ - predominantly in the stationary mode.

An analysis of both simulation characteristics of the principal components (Fig. 5) and loadings on the first eigenvector (Fig. 6) reveals presence of a special feature in system behavior at times about $100 \mathrm{~s}$. It seems obvious that degradation of simulation abilities for the first principal component is related to a more complicated structure of data set at these times. An analysis of sensor responses for the samples studied evidences that in this region there are special features for the éau-de-Cológne «Consul». In this case the responses of four sensors, namely, C[4]A, Pent, TTT and TCT, lie in a narrow (10$-15 \mathrm{~Hz}$ ) range and are characterized by multiple changes in their relative position during the first three minutes. Such a behavior is obviously determined by the peculiarities of interaction between the sensitive coatings and 


\section{I.V. Kruglenko et al.: Digital aroma technology for chemical sensing: temporal ...}

highly volatile MCM components of small molecular weights. They determine, to a great extent, specific nature of the corresponding perfume. It should be also noted that, since the QCM response is due to a mass change, low $C[4] A$ (and, to a lesser extent, Pent, TTT and TCT) responses are predominantly due to small molecular mass of the corresponding analytes for these receptors rather that absence of these analytes.

The EN-type systems are intended, first of all, for identification and classification of various MCM. Therefore the problem how to raise the discrimination ability is of primary importance. Shown in Fig. 7 is time dependence of the discrimination ability during classification analysis of three perfumes studied (we used the first principal component only and one observation for each sample). One can see that at small times it is impossible to distinguish between «Antoshka» and «Slavutich» $(\Xi>0.5)$, while at big times one cannot distinguish between «Antoshka» and «Consul» $(\Xi<-0.5)$. The point (about $100 \mathrm{~s})$ on the curve that corresponds to $\Xi=0$ determines the optimal time at which the best identification of all three samples is achieved (see Fig. $4 \mathrm{~b}$ for pictorial view). It deserves to be noted that this value is the same as the time at which the simulation abilities of the first component are the lowest. It is necessary to stress that at the same time lag bounce of observation trajectories occur. This fact may be interpreted by the change from dynamic to static regims at this time, where the best involving of process pequliarities take place. Generally this result is understandable: the most complicated data set determines both the lowest simulation and best discrimination abilities. However, the question how general this conclusion is concerning an analysis of multisensor signals still remains unanswered.

Unfortunately, the above analysis cannot enable to choose the optimal set of criteria (sensor types) to solve the classification problem of perfume type identification. To do it correctly, one has to calculate the dispersion or Fisher criteria weights and perform an analysis of their correlation to choose the corresponding orthogonal set [75]. At the same time one may evaluate the discrimination ability of certain sensor by PCA using subsets of the original data matrix: to do this coating/coatings should be eliminated from the data matrix and PCA repeated. The analysis of the calculated eigenvalues of the first eigenvector (PC1) for different subsets provides a way for estimating the role of loadings in relative importance of $\mathrm{PC} 1$ and discrimination contrast (see Table, the original data set includes $i V$-parameters calculated for threshold time $\left.t^{t h}=100 \mathrm{~s}\right)$. In the present case, sensors with $C[6] A$ and TTT coatings are absolutely essential for high discrimination ability (contrast function is always high if

Table. Relative importance of the first principal components (PC1) in the total variance in the original data set and discrimination capability (contrast $\Xi$ ) for different subsets of the original data set. Fill cells of Table (in column «Variables») correspond to variables included in the data matrix for PCA. The original data set includes $i V$-parameters calculated for threshold time $t^{\text {th }}=100 \mathrm{~s}$. Marked cells in «PC1 variance» and «Contrast $\Xi »$ columns indicate the maximum sensor contribution and the highest discrimination ability among the corresponding models.

\begin{tabular}{|c|c|c|c|c|c|c|c|c|}
\hline \multirow{2}{*}{$\begin{array}{l}\text { Model } \\
\text { Number }\end{array}$} & \multicolumn{6}{|c|}{ Variables (sensor responses included in PCA) } & \multirow{2}{*}{$\begin{array}{l}\text { PC1 variance } \\
\text { (\% of the total } \\
\text { variance) }\end{array}$} & \multirow{2}{*}{$\begin{array}{c}\text { Contrast } \Xi \\
\text { (rel.un.) }\end{array}$} \\
\hline & $C[4] A$ & $C[6] A$ & $C[8] A$ & Pent & $T T T$ & $T C T$ & & \\
\hline 1 & & & & & & & 72.9 & -0.061 \\
\hline 2 & & & & & & & 70.1 & -0.090 \\
\hline 3 & & & & & & & 71.1 & -0.215 \\
\hline 4 & & & & & & & 78.5 & -0.001 \\
\hline 5 & & & & & & & 84.0 & -0.177 \\
\hline 6 & & & & & & & 69.2 & -0.769 \\
\hline 7 & & & & & & & 72.6 & -0.017 \\
\hline 8 & & & & & & & 76 & 0.010 \\
\hline 9 & & & & & & & 80.5 & 0.610 \\
\hline 10 & & & & & & & 91.5 & -0.110 \\
\hline 11 & & & & & & & 74.0 & -0.500 \\
\hline 12 & & & & & & & 78.5 & 0.050 \\
\hline 13 & & & & & & & 91.7 & -0.079 \\
\hline 14 & & & & & & & 76.0 & 0.023 \\
\hline 15 & & & & & & & 90.4 & -0.130 \\
\hline 16 & & & & & & & 90.6 & -0.100 \\
\hline
\end{tabular}




\section{I.V. Kruglenko et al.: Digital aroma technology for chemical sensing: temporal ...}

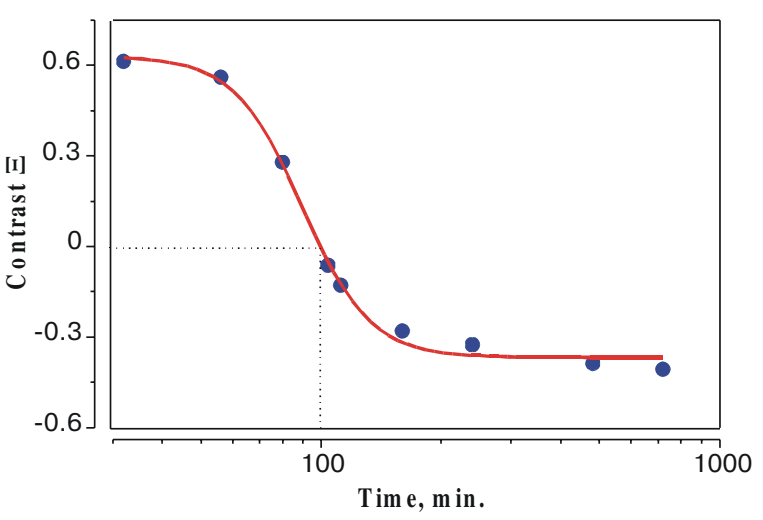

Fig. 7. Time dependence of the discrimination ability (contrast function $\Xi$, equation (7)) during classification analysis of three perfumes studied (we used the first principal component only and one observation for each sample). The «best» discrimination ability correspond to $\Xi=0$ point (see Fig. 4 for pictorial view).

sensor based on $C[6] A$ or $T T T$ is eliminated from the data matrix). This is due to the fact that the $C[6] A$ and $T T T$ coatings account for 32.6 and $33 \%$ of $\mathrm{PC} 1$ vector (or a total of $65.6 \%$ ). The striking feature in this table is that the greatest loadings similar for sensors, which are characterize by a different chemical functionality (i.e. «affinity» $C[6] A$ and «redox» $T T T$ centers).

However, the relation between discrimination ability of sensors and contribution in $\mathrm{PC} 1$, inherent loadings with high values, is not valid for the other ones. For example, the sensor with $C[8] A$ coating has not contribution in PC1. Nevertheless, the eliminating its from the data matrix (Table, Model 5) leads to greatest increasing of discrimination ability ( $\Xi$ is minimal). Thus, the sensors with small contribution in eigenvector may introduce «additional noise» in the data.

The analysis of the Models 13-15, Table, shows that the effect of sensors with middle contribution in PC1 onto both simulation and discrimination ability do not correlate directly with values of loadings ( $C[4] A-18.6$, Pent 6.4, TCT $-9.4 \%$ ). However, taking into account that both simulation and discrimination ability must be high for perfect classification analysis, the «best» subset of sensors may be defined for present case, namely, $-C[4] A$, C[6]A and TTT.

Finally, the usefulness of coating selection by using time-resolved pattern recognition are $(i)$ optimization of sensor operating mode (from kinetic point of view), (ii) reduction of array response time, (iii) decreasing number of elements in massif, (iv) increasing the discrimination ability of array for a particular application, $(v)$ possibility to predict advisability of coatings based on comparison of results of classification analysis and the types of chemical interactions that governed selectivity.

\section{Concluding remarks}

The concepts of living creatures organization and functioning serve as a source of ideas when developing and producing various technical facilities. This is most evidently seen in those areas where a profound integration of various specific lines in chemistry, physics, biology, mathematics, cybernetics and other sciences is needed to develop smart systems that imitate such high-level functions of living creatures as vision, hearing, sense of smell, etc. It is beyond doubt that for olfactronics (i.e. systems imitating the function of odor recognition) some analogies with the real «biological nose» are to be traced. Such parallels should concern both organization of the main «construction» elements and fundamentals of their functioning [76].

Here we have performed a discrimination analysis for three complex chemical compounds and illustrated how some principles (inherent in systems of odor recognition of living creatures) could be applied in science and engineering. These principles are as follows: $(i)$ separation of recognition processes and sensor response formation; (ii) chemical functionality formation in an array of low-selective sensors; (iii) time-resolving analysis of response. Application of gravitometry-type transducers enabled us to use in full measure the advantages of digital methods for signal measurement and transmission over analogous ones, as well as realize the actual separation of chemical interaction and physical detection processes in sensor elements. Separation of the three multicomponent chemical media studied has been achieved due to an allowance made for characteristic features of the human olfactory system (that are formalized in model odor systems, such as Amoore's model) when choosing sensor array elements. Concurrently an assumption has been confirmed that both affine and nucleo/ electrophilic sensing centers are of importance for system to function normally. The third important result of our studies is that one can substantially improve the discrimination ability of a multisensor array by taking into account the kinetic characteristics of system response. In this case, if one chooses, as a response parameter, an integral property that allows for both kinetic and stationary process features, then it is possible to reduce time for measurement, as well as improve the signal-to-noise ratio in the system.

The concept of development of the «electronic nose»type systems involves solution of the following principal problems: $(i)$ synthesis of receptor centers with the required chemical functionality; (ii) their integration with physical transducer; (iii) parameterization of sensor array response followed by identification based on the image recognition system; (iv) complete integration of individual system components with allowance made for the features of $(v)$ sample preparation, measurements and database formation for standard chemical images. In this case a principal problem that concerns all the stages of such systems development is search for new ways to improve both information content of each measurement and efficiency of chemical information transformation into a formalized mathematical model.

The above discussion has shown that a new technology dealing with analysis of complex multicomponent 


\section{I.V. Kruglenko et al.: Digital aroma technology for chemical sensing: temporal ...}

media should be based on temporary chemical images of the objects studied. One of the principal problems should be chemical functionality optimization for the whole array, bearing in mind possibilities of its functioning in the kinetic mode. The achievement of this objective implies the following: $(i)$ optimization of both molecular structure of receptors and ways of their immobilization on transducer; (ii) choosing both type and number of receptors that could provide the required chemical functionality of the whole array; (iii) parameterization and preliminary processing of a multidimensional sensor response to achieve the most full description of the system analyzed.

Successful realization of the above approaches will enable to develop smart sensors to monitor, both qualitatively and quantitatively, composition of gas mixtures of various natures. Such devices will be, in essence, a bioelectronic olfaction systems [77] or, in other words, will be more «real» than «electronic» nose.

\section{Acknowledgments}

We would like to thank V. Volkov for design of electronic components, V. Zhurach for device construction, O.Kopylov for assembling the sensor elements, S. Zinio for preparation the sensor coatings, V. Lyapin for paper preparation and E.Matsas for detailed assistance in pursuance of research.

\section{References}

1. A.Stephan, M.Bucking, H.Steinhart, Novel analytical tools for food flavours // Food Research International, 33(3-4), pp.199-209 (2000).

2. A.K.Pavlou, A.P.F.Turner, Sniffing out the Truth: Clinical Diagnosis Using the Electronic Nose // Clin. Chem. Lab. Med. 38(2), pp.99-112 (2000)

3. K.Morit, H.Nagao, Y.F.Sasaki, Computation of molecular information in mammalian olfactory systems // Network: Comput. Neural Syst. 9, pp.R79-R102 (1998)

4. U.Weimar, W.Goepel, Chemical Imaging: Trends in Multiparameter Sensor Systems // Proceedings: The 11th European Conference on Solid State Transducers, Warsaw, pp.527542 (1997).

5. B.M. Wise, N.L. Ricker, D.J. Veltkamp, B.R. Kowalski, A Theoretical Basis for the Use of Principal Component Models for Monitoring Multivariate Processes // Process Control \& Data 1, pp. 41-47 (1990)

6. H.L.C.Meuzelaar, J.P.Dworzanski, N.S.Arnold, W.H. McClennen, D.J.Wager, Advances in field-portable mobile GC/ MS instrumentation // Field Analytical Chemistry \& Technology, 4(1), pp.3-13 (2000).

7. C.L.Honeybourne, Organic Vapor Sensors for Food Quality Assessment // Journal of Chemical Education 77 (3), pp.338$344(2000)$

8. J.W.Gardner, Microsensors: Principles and Applications, J.Wiley\&Sons Ltd., London (1994).

9. H.Ulmer, J.Mitrovics, U.Weimar, W.Goepel, Sensor arrays with only one or several transducer principles? The advantage of hybrid modular systems // Sensors and Actuators B 65, pp.79-81 (2000)

10. G.Horner, Fragrances and aroma analysis using Electronic nose // SOFW-Journal 9, pp.1-4 (1998).

11. A.Galdikas, A.Mironas, D.Senuliene, A.Setkus, Specific set of the time constants for characterisation of organic volatile compounds in the output of metal oxide sensors // Sensors and Actuators B 68, pp.335-343 (2000).

12. R.J.Elliott-Martin, P.N.Bartlett, J.W.Gardner, T.T.Mottram, An overview of electronic noses and their applications, in Sensors and their Applications VII (ed. A.T. Augousti), IOP Publishing, Bristol, pp.12-20 (1995).

13. V.K. Varadan, J.W. Gardner, Smart tongues and smart noses, in Smart Structures and MEMS (ed. V.K. Varadan) // Proceedings of SPIE Vol. 3673, pp.67-76 (1999).

14. G.H.Dodd, P.N.Bartlett, J.W.Gardner, Odours, the stimulus for an electronic nose, in Sensors \& Sensory Systems for an Electronic Nose (eds. J.W.Gardner and P.N.Bartlett) // NATO ASI Series E: Applied Science Vol. 212 (Ch. 1), pp. 1-12 (1992).

15. B.J.Doleman, E.J.Severin, N.S.Lewis, Trends in odor intensity for human and electronic noses: Relative roles of odorant vapor pressure vs. molecularly specific odorant binding // Proc. Natl. Acad. Sci. USA 95, pp. $5442-5447$ (1998).

16. F.L.Dickert, H.Stathopulos, M.Reif, Mass-Sensitive Detection of Solvent Vapours: Predicting Sensor Effects // Adv.Mater. 8 (6), pp.525-529 (1996)

17. J.Rickert, A.Brecht, W.Goepel, QCM Operation in Liquids: Constant Sensitivity during Formation of Extended Protein Multilayers by Affinity // Anal. Chem. 69, pp. 1441-1448 (1997).

18. Y.-M.Yang, P.-Y.Yang, X.-R.Wang, Electronic nose based on SAWS array and its odour identification capability // Sensors and Actuators B 66, pp.167-170 (2000).

19. B.Jakoby, M.J.Vellekoop, Viscosity sensing using a Lovewave device // Sensors and Actuators A 68, pp. 275-281 (1998).

20. M.Haug, K.D.Schierbaum, G.Gauglitz, W.Goepel, Chemical sensors based upon polysiloxanes: comparison between optical, quartz microbalance, calorimetric, and capacitance sensors // Sensors and Actuators B 11, pp.383-391 (1993)

21. C.K.O'Sullivan, G.G.Guilbault, Commercial quartz crystal microbalances - theory and applications // Biosensors \& Bioelectronics 14, pp.663-670 (1999).

22. J.Reinbold, K.Buhlmann, K.Cammann, A.Wierig, C.Wimmer, E.Weber, Inclusion of organic vapours by crystalline hosts. Chemical-sensitive coatings for sensor applications. // Sensors and Actuators B 18-19, pp.77-81 (1994).

23. C.Fredriksson, S.Kihiman, M.Rodahl, B.Kasemo, The Piezoelectric Quartz Crystal Mass and Dissipation Sensor: A Means of Studying Cell Adhesion // Langmuir 14, pp.248251 (1998).

24. F.Hook, M.Rodah, V.P.Brzezinski, B.Kasemo, Energy Dissipation Kinetics for Protein and Antibody-Antigen Adsorption under Shear Oscillation on a Quartz Crystal Microbalance // Langmuir 14, pp.729-734 (1998).

25. J.W.Gardner, P.N.Bartlett, Electronic noses: principles and application, Oxford University Press, Oxford (1999).

26. A.J. Ricco, R.M.Crooks, G.C.Osbourn, Sensor Arrays: New Chemically Sensitive Interfaces Combined with Novel Cluster Analysis To Detect Volatile Organic Compounds and Mixtures // Acc.Chem.Res 31 (5), pp.289-295 (1998).

27. T.C.Pearce, J.W.Gardner, Predicting organoleptic scores of sub-ppm flavour notes. Part1-Part2.// The Analyst 123, pp. 2047-2063 (1998).

28. W.P.Carey, K.R.Beebe, B.R.Kowalski, Multicomponent Analysis Using an Array of Piezoelectric Crystal Sensors // Anal. Chem. 59, pp.1529-1532 (1987).

29. W.P.Carey, K.R.Beebe, B.R.Kowalski, Selection of Adsorbates for Chemical Sensor Arrays by Pattern Recognition // Anal. Chem. 58, pp.149-153 (1987).

30. J.E.Amoore, Stereochemical theory of Olfaction. // Nature 198, pp.271-272 (1964).

31. T.C.Pearce, Computational parallels between the biological olfactory pathway and its analogue 'The Electronic Nose': Part I. Biological olfaction. // BioSystems 41, pp.43-67 (1997).

32. T.Ekloev, P.Martensson, I.Lundstroem, Selection of variables for interpreting multivariate gas sensor data // Analytica Chimica Acta 381, pp. 221-232 (1999). 


\section{I.V. Kruglenko et al.: Digital aroma technology for chemical sensing: temporal ...}

33. M.Frank, T.Hemile, H.Ulmer, J.Mitrovics, U.Weimar, W.Goepel, Quality tests of electronic noses: the influence of sample dilution and sensor drifts on the pattern recognition for selected case studies // Sensors and Actuators B 65, pp.8890 (2000).

34. A.Ulmar, Organic thin films and surfaces: directions for the nineties, Thin Films, Volume 20, Academic Press, London (1995).

35. T.Weiss, K.D.Schierbaum, U.Thoden van Velzen, D.N.Reinhoudt, W.Goepel, Self-assembled monolayers of supramolecular compounds for chemical sensors // Sensors and Actuators B 26-27, pp.203-207 (1995).

36. M.A.Rodrigues, D.F.S.Petri, M.J.Politi, S.Brochsztain, Novel self-assembled films of zirconium phosphonate 1,4,5,8naphtalenediimides // Thin Solid Films 371, pp.109-113 (2000).

37. L.I.Maissel, R.Glang, Handbook of Thin Film Technology, McGraw Hill Hook Company, New York (1970).

38. E.A.Silinsh, M.V.Kurik, V.Chapek, Electronic processes in organic molecular crystals: Ljcalization and polirization phenomena, Zinatne Publishers, Riga (1988).

39. A.Rauk, Orbital interaction theory of organic chemistry, John Wiley\&Sons., Inc., Brisbane (1994).

40. F.London, Properties and application of molecular forces. I / Ztschr.Phys.Chem 11, pp.222-251 (1930).

41. V.A.Andreev, M.V.Kurik, S.Nespurek, E.A.Silinsh, V.J.Sugakov, L.F.Taure, E.L.Frankevich, V.Chapek, Electronic processes in organic molecular crystals: Transport, trapping, spin effects, Zinatne Publishers, Riga (1992).

42. A.I.Kitajigorodskji, Molecular crystals, Nauka, Moskaw (1971).

43. F.L.Dickert, O.Schuster, Supramolecular Detection of Solvent Vapours with Calixarenes: Mass-Sensitive Sensors, Molecular Mechanics and BET Studies // Mikrochim.Acta 119, pp.55-62 (1995).

44. A.Dominik, H.J.Roth, K.D.Schierbaum, W.Goepel, Supramolecular complexex based on calixarenes: force field calculations and applications for chemical sensors // Supramolecular Science 1 (1), pp.11-19 (1994).

45. O.Omar, A.K.Ray, A.K.Hassan, F.Davis, Resorcinol calixarenes (resorcarenes): Langmuir-Blodgett films and optical properties // Supramolecular Science 4 (3-4), pp.417-421 (1997).

46. G.Alberti, T.Bein, Comprehensive Supramolecular Chemistry, Elsevier Science Ltd. (1996)

47. A.S. Davidov, Biology and quantum mechanics, Naukova dumka, Kiev (1979).

48. N.Kosai, I.Sugimoto, V.Nakamura, T.Katoh, Odorant detection capability of QCR sensors coated with plasma deposited organic films // Biosensors and Bioelectronics 14, pp.533539 (1999)

49. E.G.Petrov, Physics of charge transfer in biosystems, Naukova dumka, Kiev (1984).

50. R.S. Mulliken, W.B. Person, Molecular complexes, J.Wiley, London (1969).

51. H.K.Hong, H.W.Shin, D.H.Yun, S.R.Kim, C,H,Kwon, K.Lee, T.Moriizumi, Electronic nose system with micro gas sensor array // Sensors and Actuators B 36(1-3), pp.338-341 (1996).

52. P. Michaijlov, Medicine cosmetology, Medicine, Moscow (1982).

53. V.A. Novozhilov, Odours world, Znanie, Moscow (1988).

54. R.M.Crooks, A.J.Ricco, New Organic Materials Suitable for Use in Chemical Sensor Arrays // Acc. Chem. Res. 31, pp. 219-227 (1998).

55. M.Pope, C.E.Swenberg, Electronic processes in organic crystals, Oxford University Press, Oxford (1982).

56. Ya.I.Freimanis, A.A.Urgis // Theoret. and Experim. Chem. 6, pp.678-682 (1970).

57. Ya.I.Vertsimacha, A.V.Kovalchuk, K.A.Balodis, R.C.Medne, O.Ya.Neiland, L.N.Tarachan, Optical and photoelectrical Properties of 5,6,11,12-tetrachlorotetracene // Latvijas PSR
Zintnu Akademijas Vestis, Fizikas un technisko zinatnu serija 6, pp.51-55 (1983).

58. L.Cui, M.J.Swann, A.Glidle, J.R.Barker, J.M.Cooper, Odour mapping using microresistor and piezo-electric sensor poirs / I Sensors and Actuators B 66, pp.94-97 (2000).

59. Y.Dong, G.Feng, Investigation of acoustic load sensitivity of quartz crystal resonator and related sensors // Sensors and Actuators B 66, pp.187-189 (2000).

60. R.Lucklum, P.Hauptmann, The quartz crystal microbalance: mass sensitivity, viscoelasticity and acoustic amplification // Sensors and Actuators B 70, pp.30-36 (2000).

61. C.Behling, R.Lucklum, P.Hauptmann, Response of quartzcrystal resonators to gas and liquid analyte exposure // Sensors and Actuators A 68, pp.388-398 (1998).

62. G.Sauerbrey, Verwendung von Schwingquarzen zur Waegung duenner Schichten und zur Mikrowaegung // Zeitschrift fuer Physik 155, pp. 206-222 (1959).

63. V.G.Androsova, E.G.Bronnikova, A.M.Vasiliev and all., Piezoelectric crystals, Radio i sviaz, Moscow (1992).

64. H.Nanto, N.Dougami, T.Mukai, M.Habara, E.Kusano, A.Kinbara, T.Ogawa, T.Oyabu, A smart gas sensor using polymer-film-coated quartz resonator microbalance // Sensors and Actuators B 66, pp.16-18 (2000).

65. J.W.Grade, M.Klusty, R.A.McGill, M.H.Abraham, G.Whiting, J.Andonian-Haftvan, The predomint role of swelling-induced modulus changes of the sorbent phase in determing the responses of polymer-coated surface acoustic wave vapor sensors // Anal.Chem. 64 (6), pp.610-624 (1992).

66. F.F.Volkenstein, Electronic processes at the surface of semiconductors under chemosorption, Nauka, Moscow (1987).

67. Y.M. Shirshov, B.A.Snopok, E.P.Matsas, V.I.Kal'chenko, V.V.Zhurach, A.V.Prochorovich, Y.V.Subota, I.V.Gavrilyuk, O.N.Kopylov, R.Merker, QCM-based Artificial Nose: Influence of Initial Training and Warming-up on the Responce of Thin Evaporated Calixarene Films // Proceedings of the 6th International Symposium "Olfaction\&Electronic Nose», Tuebingen. pp.130-133 (1999).

68. J.W.Gardner, T.C.Pearce, S.Friel, P.N.Bartlett, N.L.Blair, A multisensor system for beer flavour monitoring using an array of conducting polymers and predictive classifiers // Sensors and Actuators B 18-19, pp.240-243 (1994).

69. C.Di Natale, R.Paolesse, A.Macagnano, V.I.Troitsky, T.S.Berzina, A.D'Amico, Pattern recognition approach to the study of the interactions between metalloporphyrin Langmuir-Blodgett films and volatile organic compounds // Analitica Chimica Acta 384, pp.249-259 (1999).

70. J.W.Gardner, E.L.Hines, Pattern analysis techniques, in Handbook of Biosensors: Medicine, Food \& the Environment, (ed. E. Kress-Rogers), Ch. 27 pp.633-652 (1996).

71. D.M.Wilson, K.Dunman, T.Roppel, R.Kalim, Rank extraction in tin-oxide sensor arrays // Sensors and Actuators B 62(3), pp.199-210 (2000).

72. V.A.Kolemaev, O.V.Staroverov, V.B.Turundaevskji, Probability theory and mathematical statistics, Vysshaji Shkola, Moscow (1991).

73. E.Schaller, J.0.Bosset, F.Escher, Practical Experience with «Electronic Nose» Systems for Monitorinc the Quality of Dairy Products // Chimia 53, pp. 98-102 (1999).

74. M.A.Sharaf, D.L.Illman, B.R.Kowalski, Chemometrics, John Wiley and Sons, Toronto (1987).

75. B.R.Kowalski, C.F. Bender // J.Pattern Recog. 8, pp.1-12 (1976).

76. T.C.Pearce, Computational parallels between the biological olfactory pathway and its analogue «The Electronic Nose»: Part II. Sensor-based machine olfaction // BioSystems 41, pp.69-90 (1997).

77. W.Goepel, From electronic to bioelectronic olfaction, or : from artificial «moses» to real noses // Sensors and Actuators B 65, pp. 70-72 (2000). 